\title{
The Domination Entropy of the Graphs
}

\author{
Bünyamin ŞAHIN* \\ *Department of Mathematics, Faculty of Science, Selçuk University, Konya, TURKEY \\ email: bunyamin.sahin@selcuk.edu.tr
}

Keywords: Graph Entropy, Domination, Domination Polynomial

\begin{abstract}
Graph entropies have been used to interpret the complexity of the networks. There are various graph entropies in the literature. In this note a new graph entropy is defined which is based on the dominating sets of the graphs. Moreover the entropy of some classes of the graphs is obtained.
\end{abstract}

\section{Introduction}

There are many studies to determine the complexity of the networks in the last years. Computation of graph entropy measures is used in interdisciplinary researchs for example chemistry, information science and biology [1-6]. In order to determine the complexity of the graphs, most of the graph entropies are based on entropy of Shannon [7].

In the literature there are several graph entropy measures by using the order of the graphs, degree sequence of the graphs, distance of the graphs, characteristic polynomials and other graph polynomials of the graphs [7-14]. Graph entropies which are related to molecular descriptors are introduced in the last years. Calculation of graph entropy measures which are based on matchings and independent sets are defined [15] and furter studied [16]. Moreover some relations between comlexity of the graphs and Hosoya entropy are investigated Mowshowitz and Dehmer [17]. More details about the graph entropies can be found in the book [18].

As noted in [15] it could be useful to define new graph entropies based on different graph invariants. In this not a new graph entropy is introduced which is based on the dominating sets of the graphs and the entropy of some special graphs is calculated. In order to define this entropy, Dehmer's information functional approach [8] is used.

\section{Preliminaries}

Let $G$ be a simple graph with the vertex set $V(G)$ and the edge set $E(G)$. For a vertex $u \in$ $V(G)$ the notation $N_{G}(u)=\{v \mid u v \in E(G)\}$ denotes the vertices which are adjacent to $u$ and $N_{G}[u]=\{u\} \cup N_{G}(u)$. The degree of a vertex $u$ is cardinality of $N_{G}(u)$ and it is denoted by $\operatorname{deg}_{G}(u)$.

A subset $S \subseteq V(G)$ is a dominating set, if every vertex of $V \backslash S$ is adjacent to at least one vertex in $D$. The notation $\gamma(G)$ is used to show the domination number of a graph $G$ and it is cardinality of a dominating set with minimum order [19]. Domination polynomials are introduced by Alikhani and Peng [20]. Moreover domination polynomials of paths [21], cycles [22] and caterpillar graphs [23] are studied. In order to characterize the graphs, using of 
domination polynomials is an useful way. In this paper we also use domination polynomials for determining the domination entropy of the graphs.

Definition 1. The notation $\mathcal{D}(G, i)$ is used to denote the family of dominating sets of $G$ with cardinality $i$ and the notation $d_{i}$ is used to denote the cardinality of $\mathcal{D}(G, i)$ suc that $d_{i}(G)=$ $|\mathcal{D}(G, i)|$. Therefore the domination polynomial $D(G, x)$ of $G$ is introduced by the following equation [20]

$$
D(G, x)=\sum_{i=\gamma(G)}^{|V(G)|} d_{i}(G) x^{i}
$$

The entropy which are based on matchings and independent sets are computed by Hosoya and Merrifield-Simmons indices, respectively. Because total number of matchings is equal to Hosoya index and total number of independent sets is equal to Merrifield-Simmons index [15]. In here to denote the total number of dominating sets, we use $\gamma_{s}$ instead of $\gamma_{t} \cdot \gamma_{t}$ is used to denote total domination number of the graphs [19] which is a domination parameter different from the total number of the dominating sets of the graphs.

Definition 2. Let $\gamma_{s}(G)$ be the total number of dominating sets of a graph $G$. It is clear that $\gamma_{s}$ is equal to the sum of the coefficients of the domination polynomials of the graph $G$ such that

$$
\gamma_{s}(G)=\sum_{i=\gamma(G)}^{|V(G)|} d_{i}(G) .
$$

Definition 3. The entropy of a graph $G$ is defined by Dehmer's information functional approach [8] where an arbitrary information functional denoted by $f$ as follows

$$
\begin{gathered}
I_{f}(G)=-\sum_{i=1}^{|V|} \frac{f\left(v_{i}\right)}{\sum_{j=1}^{|V|} f\left(v_{j}\right)} \log \left(\frac{f\left(v_{i}\right)}{\sum_{j=1}^{\lceil V\rceil} f\left(v_{j}\right)}\right) \\
=\log \left(\sum_{i=1}^{|V|} f\left(v_{i}\right)\right)-\sum_{i=1}^{|V|} \frac{f\left(v_{i}\right)}{\sum_{j=1}^{[V]} f\left(v_{j}\right)} \log f\left(v_{i}\right) .
\end{gathered}
$$

Now we can give the definiton of the domination entropy by using a new information funtional.

Definition 4. For a graph $G$ with $|V|=n$, we introduce the information functional such that

$$
f:=d_{i}(G)
$$

with $d_{i}(G)=|\mathcal{D}(G, i)|$. Thus for each $i<\gamma(G) d_{i}(G)=0, d_{n-1}(G)=n$ and $d_{n}(G)=1$.

Then by using Definition 3 we obtain the domination entropy

$$
I_{d}(G)=I_{f}(G)=-\sum_{i=1}^{n} \frac{d_{i}(G)}{\gamma_{s}(G)} \log \left(\frac{d_{i}(G)}{\gamma_{s}(G)}\right)
$$




$$
\begin{gathered}
=\log \left(\gamma_{s}(G)\right)-\frac{1}{\gamma_{a}(G)} \sum_{i=1}^{n} d_{i}(G) \log \left(d_{i}(G)\right) \\
=\log \left(\gamma_{s}(G)\right)-\frac{1}{\gamma_{s}(G)} \sum_{i=1}^{n-2} d_{i}(G) \log \left(d_{i}(G)\right)-\frac{n \log n}{\gamma_{s}(G)}
\end{gathered}
$$

\section{Domination Entropy of Some Graphs}

We first give an essential theorem about the entropy of a graph which is the union of two connected graphs.

Theorem 1. Assume that $H_{1}$ and $H_{2}$ are two connected graphs and $H=H_{1} \cup H_{2}$ is the disjoint union of $H_{1}$ and $H_{2}$. We obtain that

$$
I_{d}(G)=\log \left(\sum_{i=\gamma(H)}^{n} d_{i}(H)\right)-\frac{1}{\sum_{i=\gamma(H)}^{n} d_{i}(H)} \sum_{i=1}^{n} d_{i}(H) \log \left(d_{i}(H)\right)
$$

such that $\gamma(H)=\gamma\left(H_{1}\right)+\gamma\left(H_{2}\right)$ and $d_{i}(H)=\sum_{j=\gamma\left(H_{1}\right)}^{i} d_{j}\left(H_{1}\right) d_{i-j}\left(H_{2}\right)$.

For a graph $G$, we take the number of vertices $n$ and $d_{n-1}(G)=n$ and $d_{n}(G)=1$ in this section.

Theorem 2. For a complete graph $K_{n}$ of order $n$, we obtain that

$$
I_{d}\left(K_{n}\right)=\log \left(2^{n}-1\right)-\frac{1}{2^{n}-1} \sum_{i=1}^{n}\left(\begin{array}{c}
n \\
i
\end{array}\right) \log \left(\left(\begin{array}{c}
n \\
i
\end{array}\right)\right) .
$$

Proof. Let $G$ be a complete graph $K_{n}$. Since $\gamma(G)=1$, there exists a dominating set of $G$ for each cardinality $1 \leq i \leq n$ with $d_{i}(G)=\left(\begin{array}{c}n \\ i\end{array}\right)$. Thus $\gamma_{s}(G)=2^{n}-1$ containing the all subsets of $V(G)$ except that empty set. Therefore

$$
I_{d}\left(K_{n}\right)=\log \left(2^{n}-1\right)-\frac{1}{2^{n}-1} \sum_{i=1}^{n}\left(\begin{array}{l}
n \\
i
\end{array}\right) \log \left(\left(\begin{array}{c}
n \\
i
\end{array}\right)\right) .
$$

Theorem 3. For a star graph $S_{n}$ with $n$ vertices we obtain the domination entropy

$$
I_{d}\left(S_{n}\right)=\log \left(2^{n-1}+1\right)-\frac{1}{2^{n-1}+1}\left(\sum_{i=1}^{n-3}\left(\begin{array}{c}
n-1 \\
i
\end{array}\right) \log \left(\left(\begin{array}{c}
n-1 \\
i
\end{array}\right)\right)\right)-\frac{n \log n}{2^{n-1}+1}
$$

Proof. Let $G$ be a complete graph $S_{n}$. We know that domination polynomial of the $S_{n}$ is [20] 


$$
D\left(S_{n}, x\right)=x^{n-1}+x(1+x)^{n-1} .
$$

Since $\gamma\left(S_{n}\right)=1$, by Definition 2 we obtain that

$$
\gamma_{s}\left(S_{n}\right)=\sum_{i=1}^{n} d_{i}(G)=2^{n-1}+1
$$

Now we can determine the number of the dominating sets with cardinality $i$. The star graph $S_{n}$ have a vertex $u$ which has degree $(n-1)$ and the other $(n-1)$ vertices have degree one. In order to dominate the graph $S_{n}$, the vertex $u$ is contained by every dominating set except that a set. The only dominating set of the $S_{n}$ which has cardinality $(n-1)$ does not contain $u$ and it is consisted of the vertices with degree one.

Therefore the set with cardinality one is $\{u\}$ and $d_{1}(G)=1$.

The sets with cardinality two are consisted of $u$ and a vertex whose degree is one. Thus $d_{2}(G)=$ $n-1, d_{3}(G)=\left(\begin{array}{c}n-1 \\ 2\end{array}\right), \ldots, d_{n-1}(G)=\left(\begin{array}{c}n-1 \\ 1\end{array}\right)+1=n$ and $d_{n}(G)=1$. Consequently we obtain that

$$
I_{d}\left(S_{n}\right)=\log \left(2^{n-1}+1\right)-\frac{1}{2^{n-1}+1}\left(\sum_{i=1}^{n-3}\left(\begin{array}{c}
n-1 \\
i
\end{array}\right) \log \left(\left(\begin{array}{c}
n-1 \\
i
\end{array}\right)\right)\right)-\frac{n \log n}{2^{n-1}+1}
$$

Theorem 4. For a double star graph $S_{a, b}$ we obtain the domination entropy

$$
\begin{gathered}
I_{d}\left(S_{a, b}\right)=\log \left(\gamma_{s}\left(S_{a, b}\right)\right)-\frac{1}{\gamma_{s}\left(S_{a, b}\right)}\left(\sum_{i=2}^{a+b-3} d_{i}(\mathrm{G}) \log \left(d_{i}(\mathrm{G})\right)\right) \\
-\frac{\left(\left(\begin{array}{c}
a+b-2 \\
2
\end{array}\right)+a+b-1\right) \log \left(\left(\begin{array}{c}
a+b-2 \\
2
\end{array}\right)+a+b-1\right)+(a+b) \log (a+b)}{\gamma_{s}\left(S_{a, b}\right)} .
\end{gathered}
$$

Proof. Let $G$ be a double star graph $S_{a, b}$. Assume that $a \leq b$. We know that domination polynomial of the $S_{a, b}$ is [23]

$$
D\left(S_{a, b}, x\right)=x^{2}(1+x)^{a+b-2}+x\left(x^{a-1}(1+x)^{b-1}+x^{b-1}(1+x)^{a-1}\right)+x^{a+b-2} .
$$

Note that $\gamma\left(S_{a, b}\right)=2$. By Definition 2 we obtain that

$$
\gamma_{s}\left(S_{a, b}\right)=\sum_{i=2}^{n} d_{i}(G)=2^{a+b-2}+2^{a-1}+2^{b-1}+1 .
$$

We determine the number of the dominating sets with cardinality $i$. The star graph $S_{a, b}$ have two vertices $u$ and $v$ which have degree $a$ and $b$, respectively. The remaining $(a+b-2)$ vertices have degree one. In order to dominate the graph $S_{a, b}$, there are three cases. These cases are, the vertices $u, v$ are contained by dominating sets, one of the $u$ and $v$ is contained in dominating sets and none of the $u$ and $v$ is contained in dominating sets [23]. For the last case 
the only dominating set of the $S_{a, b}$ does not contain the vertices $u$ and $v$ and it is consisted of the vertices with degree one.

Therefore the set with cardinality two is $\{u, v\}$ and $d_{2}(G)=1$.

The sets with cardinality three are consisted of $u, v$ and a vertex whose degree is one. Thus $d_{3}(G)=a+b-2$. By this way we obtain the dominating sets of the $S_{a, b}$ such that

$$
\begin{gathered}
d_{4}(G)=\left(\begin{array}{c}
a+b-2 \\
2
\end{array}\right), \ldots, d_{a}(G)=\left(\begin{array}{c}
a+b-2 \\
a-2
\end{array}\right)+1, d_{a+1}(G)=\left(\begin{array}{c}
a+b-2 \\
a-1
\end{array}\right)+\left(\begin{array}{c}
b-1 \\
1
\end{array}\right), \\
d_{a+2}(G)=\left(\begin{array}{c}
a+b-2 \\
a
\end{array}\right)+\left(\begin{array}{c}
b-1 \\
2
\end{array}\right), \ldots, d_{b-1}(G)=\left(\begin{array}{c}
a+b-2 \\
b-3
\end{array}\right)+\left(\begin{array}{l}
b-1 \\
b-a
\end{array}\right), \\
d_{b}(G)=\left(\begin{array}{c}
a+b-2 \\
b-2
\end{array}\right)+1+\left(\begin{array}{l}
b-1 \\
b-a
\end{array}\right), \ldots, \\
d_{a+b-2}(G)=\left(\begin{array}{c}
a+b-2 \\
a+b-4
\end{array}\right)+\left(\begin{array}{c}
a-1 \\
a-2
\end{array}\right)+\left(\begin{array}{l}
b-1 \\
b-2
\end{array}\right)+1 \\
d_{a+b-1}(G)=\left(\begin{array}{c}
a+b-2 \\
a+b-3
\end{array}\right)+2=a+b, d_{a+b}(G)=1 .
\end{gathered}
$$

We can generalize the cardinality of the dominating sets with cardinality $d_{i}(G)$ for $(n-3) \geq$ $i \geq 2$ such that $3 \leq j \leq a+b-2$. We accept that $\left(\begin{array}{l}k \\ l\end{array}\right)=0$ for $k<l$.

$$
d_{(a+b)-j}(G)=\left(\begin{array}{c}
a+b-2 \\
j
\end{array}\right)+\left(\begin{array}{l}
a-1 \\
a-j
\end{array}\right)+\left(\begin{array}{l}
b-1 \\
b-j
\end{array}\right)
$$

Finally we obtain that

$$
\begin{gathered}
I_{d}\left(S_{a, b}\right)=\log \left(\gamma_{s}\left(S_{a, b}\right)\right)-\frac{1}{\gamma_{s}\left(S_{a, b}\right)}\left(\sum_{i=2}^{a+b-3} d_{i}(\mathrm{G}) \log \left(d_{i}(\mathrm{G})\right)\right) \\
-\frac{\left(\left(\begin{array}{c}
a+b-2 \\
2
\end{array}\right)+a+b-1\right) \log \left(\left(\begin{array}{c}
a+b-2 \\
2
\end{array}\right)+a+b-1\right)+(a+b) \log (a+b)}{\gamma_{s}\left(S_{a, b}\right)} .
\end{gathered}
$$

Theorem 5. For a complete bipartite graph $K_{a, b}$ with cardinality $n$ we obtain the domination entropy

$$
I_{d}\left(K_{a, b}\right)=\log \left(\gamma_{s}\left(K_{a, b}\right)\right)
$$




$$
\begin{aligned}
& -\frac{1}{\gamma_{s}\left(K_{a, b}\right)}\left(\sum_{\substack{i=2 \\
i \neq a, b}}^{n} \sum_{j=1}^{i-1}\left(\begin{array}{c}
a \\
j
\end{array}\right)\left(\begin{array}{c}
b \\
i-j
\end{array}\right) \log \left(\sum_{\substack{i=2 \\
i \neq a, b}}^{n} \sum_{j=1}^{i-1}\left(\begin{array}{l}
a \\
j
\end{array}\right)\left(\begin{array}{c}
b \\
i-j
\end{array}\right)\right)+d_{a}(G) \log \left(d_{a}(G)\right)\right. \\
& \left.+d_{b}(G) \log \left(d_{b}(G)\right)\right)
\end{aligned}
$$

Proof. We know that domination polynomial of the $K_{a, b}$ is [20] with $n=a+b$,

$$
D\left(K_{a, b}, x\right)=\left((1+x)^{a}-1\right)\left((1+x)^{b}-1\right)+x^{a}+x^{b} .
$$

Moreover the partite sets of $K_{a, b}$ is consisted of two sets with $a$ vertices and $b$ vertices. Thus we use the domination number of $K_{a, b}$ with $\gamma\left(K_{a, b}\right)=2$ with chosen vertices, a vertex from $a$ vertices and a vertex from $b$-vertices. By Definition 2 we obtain that

$$
\gamma_{s}\left(K_{a, b}\right)=\sum_{i=2}^{n} d_{i}(G)=2^{a+b}-2^{a}-2^{b}+3 .
$$

Assume that $a \leq b$. Now we determine the number of dominating sets of the $K_{a, b}$ such that

$$
\begin{gathered}
d_{2}(G)=\left(\begin{array}{l}
a \\
1
\end{array}\right)\left(\begin{array}{l}
b \\
1
\end{array}\right)=a b, \\
d_{3}(G)=\left(\begin{array}{l}
a \\
1
\end{array}\right)\left(\begin{array}{l}
b \\
2
\end{array}\right)+\left(\begin{array}{l}
a \\
2
\end{array}\right)\left(\begin{array}{l}
b \\
1
\end{array}\right), \ldots, \\
d_{a}(G)=\sum_{i=1}^{a}\left(\begin{array}{l}
a \\
i
\end{array}\right)\left(\begin{array}{c}
b \\
a-i
\end{array}\right)=\left(\begin{array}{l}
a \\
1
\end{array}\right)\left(\begin{array}{c}
b \\
a-1
\end{array}\right)+\cdots+\left(\begin{array}{c}
a \\
a-1
\end{array}\right)\left(\begin{array}{l}
b \\
1
\end{array}\right)+1, \ldots, \\
d_{b}(G)=\sum_{j=1}^{b}\left(\begin{array}{c}
a \\
b-j
\end{array}\right)\left(\begin{array}{l}
b \\
j
\end{array}\right)=\left(\begin{array}{c}
a \\
b-1
\end{array}\right)\left(\begin{array}{l}
b \\
1
\end{array}\right)+\cdots+\left(\begin{array}{c}
a \\
1
\end{array}\right)\left(\begin{array}{c}
b \\
b-1
\end{array}\right)+1 .
\end{gathered}
$$

By this way we obtain number of the last two dominating sets

$$
d_{n-1}(G)=\left(\begin{array}{c}
a \\
a-1
\end{array}\right)\left(\begin{array}{l}
b \\
b
\end{array}\right)+\left(\begin{array}{l}
a \\
a
\end{array}\right)\left(\begin{array}{c}
b \\
b-1
\end{array}\right)=a+b
$$

and $d_{a+b}(G)=1$.

Finally we obtain the domination entropy of the complete bipartite graph $K_{a, b}$

$$
I_{d}\left(K_{a, b}\right)=\log \left(\gamma_{s}\left(K_{a, b}\right)\right)
$$




$$
\begin{aligned}
& -\frac{1}{\gamma_{s}\left(K_{a, b}\right)}\left(\sum_{\substack{i=2 \\
i \neq a, b}}^{n} \sum_{j=1}^{i-1}\left(\begin{array}{l}
a \\
j
\end{array}\right)\left(\begin{array}{c}
b \\
i-j
\end{array}\right) \log \left(\sum_{\substack{i=2 \\
i \neq a, b}}^{n} \sum_{j=1}^{i-1}\left(\begin{array}{c}
a \\
j
\end{array}\right)\left(\begin{array}{c}
b \\
i-j
\end{array}\right)\right)+d_{a}(G) \log \left(d_{a}(G)\right)\right. \\
& +d_{b}(G) \log \left(d_{b}(G)\right)
\end{aligned}
$$

\section{Conclusion}

In this paper we defined domination entropy by using information functionals with the dominating sets of the graphs. In future works we compare the domination entropy of the graphs with the entropies based on the mathchings and independent sets. Furthermore total number of matchings and total number of independent sets are well known topological indices in graph theory as Hosoya index and Merrifield-Simmons index. The total number of dominating sets is not studied as a topological index to our best knowledge. It is an interesting topic, investigation of the number of dominating sets of the graphs which can be called as 'Domination Index'.

\section{References}

[1] V. Kraus, M. Dehmer, F. Emmert-Streib, Probabilistic inequalities for evaluating structural network measures, Inform. Sci. 288 (2014) 220-245.

[2] M. Dehmer, F. Emmert-Streib, M. Grabner, A computational approach to construct a multivariate complete graph invariant, Inform. Sci. 260 (2014) 200-208.

[3] A. Mowshowitz, M. Dehmer, Entropy and the complexity of graphs revisited, Entropy 14 (3) (2012) 559-570.

[4] M. Dehmer, A. Mowshowitz, A case study of cracks in the scientific enterprise: reinvention of information-theoretic measures for graphs, Complexity (2014).

[5] Y. Chen, K. Wu, X. Chen, C. Tang, Q. Zhu, An entropy-based uncertainty measurement approach in neighborhood systems, Inform. Sci. 279 (2014), 239-250.

[6] C. Wang, A. Qu, Entropy, similarity measure and distance measure of vague soft sets and their relations, Inform. Sci. 244 (2013) 92-106.

[7] M. Dehmer, A. Mowshowitz, A history ofgraph entropy measures, Inform. Sci. 181 (2011) 57-78.

[8] M. Dehmer, Information processing in complex networks: graph entropy and information functionals, Appl. Math. Comput. 201 (2008) 82-94.

[9] M. Dehmer, A. Mowshowitz, Y. Shi, Structural differentiation of graphs using Hosoyabased indices, PLoS ONE 9 (7) (2014) e102459.

[10] S. Dragomir, C. Goh, Some bounds on entropy measures in information theory, Appl. Math. Lett. 10 (1997) 23-28.

[11] Z. Chen, M. Dehmer, Y. Shi, A note on distance-based graph entropies, Entropy 16 (10) (2014) 5416-5427. 
[12] J. Cao, M. Shi, L. Feng, On the edge-hyper-hamiltonian laceability of balanced hypercubes, Discuss Math. Graph Theory. 36 (2016) 805-817.

[13] L. Feng, J. Cao, W. Liu, S. Ding, H. Liu, The spectral radius of edge chromatic critical graphs, Linear Algebra Appl. 492 (2016) 78-88.

[14] G. Yu, X. Liu, H. Qu, Singularity of Hermitian (quasi-)Laplacian matrix of mixed graphs, Appl. Math. Comput. 293 (2017) 287-292.

[15] S. Cao, M. Dehmer, Z. Kang, Network entropies based on independent sets and matchings, Appl. Math. Comput. 307 (2017) 265-270.

[16] P. Wan, X. Chen, J. Tu, M. Dehmer, S. Zhang, F. Emmert-Streib, On graph entropy measures based on the number of independent sets and matchings Inform. Sci. 516 (2020) 491 504.

[17] A. Mowshowitz, M. Dehmer, The Hosoya entropy of a graph, Entropy 17 (2015) 10541062.

[18] M. Dehmer, Z. Chen, X. Li, Y. Shi, F. Emmert-Streib, Mathematical Foundations and Applications of Graph Entropy, Wiley-Blackwell, 2016.

[19] T.W. Haynes, S.T. Hedetniemi, P.J. Slater, Fundamentals of Domination in Graphs, Marcel Dekker, New York, 1998.

[20] S. Alikhani, Y.H. Peng, Introduction to domination polynomial of a graph, Ars Combin. 114 (2014), 257-266.

[21] S. Alikhani, Y.H. Peng, Dominating sets and domination polynomial of paths, Int. J Math. Math. Sci., Vol 2009, Article ID 542040 (2009).

[22] S. Alikhani, Y.H. Peng, Dominating sets and domination polynomial of cycles, Global Journal of Pure and Applied Mathematics 4 (2) (2008) , 151-162

[23] B. Şahin, A. Şahin, On domination polynomials of caterpillar graphs, Turkish J. Math. Comp. Sci. 9 (2018), 34-38. 